\title{
Accuracy of the GRACE and TIMI Scores in Predicting the Angiographic Severity of Acute Coronary Syndrome
}

\author{
Carolina Esteves Barbosa, Mateus Viana, Mariana Brito, Michael Sabino, Guilherme Garcia, Mayara Maraux, \\ Alexandre Costa Souza, Márcia Noya-Rabelo, J. Péricles Esteves, Luis Cláudio Lemos Correia \\ Escola Bahiana de Medicina e Saúde Pública; Hospital São Rafael; Hospital Português, Salvador, BA - Brazil
}

\begin{abstract}
Background: The accuracy of the GRACE and TIMI scores in predicting coronary disease extension in patients with non-ST-elevation acute coronary syndromes (ACS) has not been established.

Objective: To assess the hypothesis that the GRACE and TIMI risk scores satisfactorily predict coronary disease extension in patients with non-ST-elevation ACS undergoing coronary angiography.

Methods: Individuals meeting the objective criteria for ACS and undergoing coronary angiography during hospitalization were consecutively assessed. Angiographic coronary disease was described as follows: quantification of coronary disease extension by using Gensini score; presence of any coronary artery obstruction ( $\geq 70 \%$ or $\geq 50 \%$ when affecting left main coronary artery); and presence of severe disease (three-vessel disease or affecting the left main coronary artery).
\end{abstract}

Results: Of 112 patients assessed, a positive correlation of the Gensini score was observed with the GRACE $(p=0.017)$ and TIMI $(p=0.02)$ scores, but that association was weak $(r=0.23$ and $r=0.27$; respectively). The GRACE SCore could predict neither obstructive coronary disease (area under the ROC curve $=0.57 ; 95 \% \mathrm{Cl}=0.46-0.69$ ), nor severe coronary disease $(\mathrm{ROC}=\mathbf{0 . 5 9} ; 95 \% \mathrm{CI}=\mathbf{0 . 4 8}-\mathbf{0 . 7 0})$. The TIMI score proved to be a modest predictor of coronary disease $(\mathrm{ROC}=0.65 ; 95 \% \mathrm{Cl}=0.55-0.76)$ and of severe coronary disease $(\mathrm{ROC}=0.66 ; 95 \% \mathrm{CI}=0.56-0.76)$.

Conclusion: (1) There is a positive association between the values of the TIMI or GRACE scores and the extension of coronary artery disease in patients with ACS; $(2)$ however, the degree of that association is not sufficient to make those scores accurate predictors of coronary angiography results. (Arq Bras Cardiol 2012;99(3):818-824)

Keywords: Acute coronary syndrome / complications; propensity score; coronary angiography / utilization; coronary vessels / anatomy \& histology.

\section{Introduction}

Individuals hospitalized due to non-ST-elevation acute coronary syndromes (ACS) have a wide variation in their disease severity, which ranges according to clinical and laboratory characteristics ${ }^{1}$. Thus, risk stratification is essential for adequate clinical decision, discriminating individuals who benefit from more aggressive strategies. The use of multivariate models in the form of scores has been shown to represent the most accurate way to predict risk, being superior to the subjective clinical impression ${ }^{2}$. The TIMI and GRACE risk scores are the most used, and their prognostic value has been established by prospective cohort studies ${ }^{1,3}$.

In addition to prognostic assessment, predicting the anatomical extension of coronary artery disease is potentially useful for clinical decision. This is due to the fact that the prediction of obstructive disease (or severely obstructive

Mailing Address: Luis Cláudio Lemos Correia • Av. Princesa Leopoldina, 19/402, Graça. Postal Code 40150-080, Salvador, BA - Brazil

E-mail: Iccorreia@cardiol.br; Iccorreia@terra.com.br

Manuscript received November 16, 2011; manuscript revised November 16 accepted April 5, 2012. disease) represents one more criterion in favor of performing coronary angiography, because that finding anticipates the need for myocardial revascularization. Some studies have shown an association between the TIMI score value and the number of arteries affected by obstructive disease. However, none of those studies has performed an analysis of discriminatory accuracy, and, thus, the diagnostic value of that score regarding the presence of coronary artery disease and its severity remains uncertain ${ }^{4-7}$. Regarding the GRACE score, no study has assessed its association with coronary artery anatomy.

Aiming at testing the hypothesis that the TIMI and GRACE risk scores are accurate predictors of the presence and extension of obstructive coronary artery disease, this study analyzed patients consecutively admitted to the Acute Coronary Syndromes Registry (Resca), who underwent coronary angiography and had not undergone revascularization surgery. The predictive capacity of the scores was analyzed regarding the following: the quantitative measure of coronary artery disease extension (Gensini score); the presence of any obstructive coronary artery disease; and the presence of severe obstructive coronary artery disease. 


\section{Methods}

\section{Sample selection}

Individuals consecutively admitted to the Coronary Unit of our hospital from August 2007 to January 2009, diagnosed with unstable angina or non-ST-segment elevation myocardial infarction, were considered candidates to Resca. The inclusion criterion in that registry has been defined as typical chest discomfort at rest for the past 48 hours, associated with at least one of the following characteristics: 1) positive myocardial necrosis marker, defined as troponin $\mathrm{T} \geq 0.01 \mathrm{ug} / \mathrm{L}$ or troponin I > $0.034 \mu \mathrm{g} / \mathrm{L}$, corresponding to values over the $99^{\text {th }}$ percentile $\left.{ }^{8,9} ; 2\right)$ ischemic electrocardiographic changes, consisting of $\mathrm{T}$ wave inversion $(\geq 0.1 \mathrm{mV})$ or transient STsegment depression ( $\geq 0.05 \mathrm{mV}) ; 3$ ) coronary artery disease previously documented, defined by a history of myocardial infarction or previous angiography showing coronary artery obstruction $\geq 50 \%$. For the current analysis, of the patients included in Resca, those undergoing invasive coronary angiography during hospitalization were selected.

\section{GRACE and TIMI scores}

For calculating the scores, the following data were used: clinical data on the occasion of patient's presentation to the emergency service; electrocardiographic records of the first six hours of medical care; troponin T measures regarding the first 12 hours of medical care; and the first plasma creatinine value. Elevation of the myocardial necrosis marker as a component of the scores was defined as troponin $\mathrm{T} \geq 0.01 \mathrm{ug} / \mathrm{L}$ or troponin $\mathrm{I} \geq 0.034 \mu \mathrm{g} / \mathrm{L}$, that is, above the $99^{\text {th }}$ percentile ${ }^{8,9}$. Killip classification ${ }^{10}$ has also been applied to patients with unstable angina so that the GRACE score could be calculated.

The criteria previously defined in the respective validation studies of those scores were used. Briefly, the TIMI risk score consists of seven dichotomous variables. The presence of each variable adds one point to the total score, which ranges from zero to seven. Such variables relate to the clinical presentation of ACS (ST-segment depression, elevation in myocardial necrosis marker, more than one angina episode in 24 hours) or the previous characteristics of the patients (age $\geq 65$ years, use of aspirin, coronary artery obstruction $\geq 50 \%$, at least three risk factors for atherosclerotic disease) ${ }^{1}$.

The GRACE score consists of eight variables, five of which are computed semi-quantitatively, that is, with a different weight for each stratum of age, systolic blood pressure, heart rate, plasma creatinine and Killip class; the other three are computed dichotomously (ST-segment depression, elevation in myocardial necrosis marker, cardiac arrest on admission). The final score can range from zero to $372^{3}$.

\section{Assessment of coronary artery disease extension}

Coronary artery anatomy was assessed by one single experienced cardiac catheterization physician, who defined the coronary artery disease extension quantitatively and categorically. Quantitative analysis was performed by using the Gensini score ${ }^{11,12}$. Briefly, that score assesses 28 coronary artery segments, which are scored according to their anatomical importance (ranging from 0.5 to 5 ) multiplied by the score regarding the maximum degree of obstruction $(0-25 \%=2$; $26 \%-50 \%=4 ; 51 \%-75 \%=8 ; 76 \%-90 \%=16 ; 91 \%-99 \%=$ $32 ; 100 \%=64$ points). The points of the 28 segments are summed to yield a final score.

From the categorical viewpoint, coronary artery anatomy was defined as follows: presence of obstructive coronary artery disease (any obstruction $\geq 70 \%$ or $\geq 50 \%$ if in the left main coronary artery); presence of severe coronary artery disease (obstruction $\geq 70 \%$ in the anterior descending, circumflex and right coronary artery, characterizing a three-vessel pattern, or obstruction $\geq 50 \%$ in the left main coronary artery).

\section{Data analysis}

Considering that the Gensini score showed non-normal distribution (Kolmogorov-Smirnov test), the statistical analyses were mainly non-parametric. Aiming at assessing the association between the GRACE or TIMI risk scores and coronary artery disease extension, some analyses were used. First, the linear association of each risk score with the Gensini score was assessed by use of Spearman correlation, while linear regression was used to demonstrate the strength of the influence of the GRACE and TIMI score values on coronary artery disease extension. Second, the Kruskal-Wallis test was used to compare the Gensini score values between the groups divided according to the tertiles of risk scores. Third, the receiver-operating characteristics (ROC) curve was used to test the predictive accuracy of risk scores regarding the presence of coronary artery disease and of severe coronary artery disease. Significant prediction occurred when the area under the ROC curve was statistically different from 0.5.

The sample size necessary for the correlation analyses and description of the areas under the ROC curve was estimated. For the first analysis, considering a minimum correlation coefficient of 0.25 and alpha of 0.05 , a sample size of 96 patients would suffice to provide a statistical power of $80 \%$ in the null hypothesis rejection of lack of correlation. In the second analysis, considering an area under the ROC curve of $0.65,100$ patients ( 30 without disease and 70 with obstructive disease or 70 without severe disease and 30 with severe disease) would be necessary to provide a precision of \pm 0.12 in the confidence interval $(\mathrm{Cl})$ of the ROC curve, which would suffice to reject the null hypothesis of the 0.50 area.

The scores were described as median and interquartile interval (IQI), and statistical significance was defined as $\mathrm{p}<0.05$. The SPSS Statistical Software (9.0 version, SPSS Inc. Chicago, Illinois, USA) was used for data analysis.

\section{Results}

\section{Characteristics of the sample}

During the study, 241 patients were admitted to the Resca, 76 of whom did not undergo coronary angiography during hospitalization and 53 had already undergone surgical myocardial revascularization. Thus, this study assessed 112 patients (mean age of $70 \pm 12$ years; $51 \%$ of the male sex), $63 \%$ of whom diagnosed with non-ST-elevation acute myocardial infarction and the others with unstable angina. The 


\section{Original Article}

median of the GRACE score was 115 (IQI: 91 - 141), while that of the TIMI score was 3 (IQI: 2 - 4), indicating a symmetrical distribution of individuals in the low-, intermediate- and high-risk groups. Killip class $>1$ and systolic dysfunction at least moderate (ejection fraction $<45 \%$ ) were identified in only $11 \%$ and $12 \%$ of the patients, respectively. Other clinical characteristics are shown in Table 1.

The analysis of the coronary angiography showed that $71 \%$ of the patients had at least one coronary artery obstruction $\geq 70 \%$. Severe coronary artery disease (three-vessel disease or left main coronary artery) was identified in $32 \%$ of the sample, two-vessel disease in $18 \%$ of the sample, single-vessel disease in $21 \%$, and no coronary artery obstruction in $29 \%$ of the sample. The Gensini angiographic score showed median of 103 (IQI: 65 - 155) and non-normal distribution ( $p<0.001)$.

\section{Coronary artery anatomy prediction by use of the GRACE score}

Positive linear association was observed between the GRACE and Gensini scores, represented by the regression formula Gensini $=48+0.65 \times$ GRACE $(p=0.001)$. Although that relationship is statistically significant, the Spearman correlation coefficient indicated a weak association $(r=0.23$; $\mathrm{p}=0.017)$ - Figure 1A.

The Gensini score had a median of 85 (IQI: 60 - 128) in the first tertile of the GRACE score, a median of 89 (67 - 167) in the second tertile, and a median of $132(78-187)$ in the third tertile. This tendency towards greater coronary artery disease extension according to the GRACE tertiles did not reach statistical significance $(p=0.099)$ - Figure 1B.

Despite those tendencies, the GRACE score showed no discriminatory capacity between patients with and without obstructive coronary artery disease, having an area under the ROC curve of 0.57 (95\% Cl: $0.46-0.69 ; p=0.22)$ - Figure $1 \mathrm{C}$. The prevalence of obstructive coronary artery disease was similar in the first, second and third tertiles of the GRACE score $(66 \%, 71 \%$, and $77 \%$, respectively; $p=0.58)$. Similarly, the GRACE score could not discriminate patients with and without severe coronary artery disease (left main coronary artery or three-vessel disease) - area under the ROC curve of 0.59 $(95 \% \mathrm{Cl}: 0.48-0.70 ; p=0.13)-$ Figure 1D. The prevalence of severe coronary artery disease did not significantly differ between the three GRACE tertiles (25\%, 33\%, and 37\%, respectively; $\mathrm{p}=0.56$ ).

\section{Prediction of coronary artery anatomy by use of the TIMI score}

Positive linear association was observed between the TIMI and Gensini scores, represented by the regression formula Gensini $=79+16 \times \mathrm{TIMI}(\mathrm{p}=0.009)$. Although that relationship is statistically significant, the Spearman correlation coefficient indicated a weak association $(r=0.28 ; p=0.003)$ - Figure 2A.

The Gensini score had a median of 78 (IQI: $62-117)$ in the first tertile of the TIMI score, a median of 107 (62 - 188) in the second tertile, and a median of 120 (95 - 166) in the third tertile $(p=0.024)$ - Figure $2 B$.

The TIMI score showed a modest discriminatory capacity between patients with and without obstructive coronary artery disease, having an area under the ROC curve of 0.65 (95\%
Table 1 - Characteristics of the sample

\begin{tabular}{|c|c|}
\hline \multicolumn{2}{|l|}{ Clinical characteristics } \\
\hline Sample & 112 \\
\hline Age (years) & $70 \pm 12$ \\
\hline Males & $57(51 \%)$ \\
\hline Diabetes & $35(31 \%)$ \\
\hline ST-segment depression & $17(15 \%)$ \\
\hline Troponin-positive & $70(63 \%)$ \\
\hline Serum creatinine (mg/dL) & $1.24 \pm 1.04$ \\
\hline Systolic blood pressure $(\mathrm{mm} \mathrm{Hg})$ & $152 \pm 33$ \\
\hline Heart rate (bpm) & $79 \pm 22$ \\
\hline Killip $>1$ & $12(11 \%)$ \\
\hline LV ejection fraction < $45 \%$ & $12 / 101(12 \%)$ \\
\hline Obstructive CAD & $80(71 \%)$ \\
\hline Three-vessel or LMC disease & $36(32 \%)$ \\
\hline GRACE score & $115(91-141)$ \\
\hline TIMI score & $3(2-4)$ \\
\hline Gensini score & $103(65-155)$ \\
\hline \multicolumn{2}{|l|}{ In-hospital treatment } \\
\hline Aspirin & $110(98 \%)$ \\
\hline Clopidogrel & $106(95 \%)$ \\
\hline GP Ilb/llla blocker & $6(5.3 \%)$ \\
\hline Heparins & $103(92 \%)$ \\
\hline Statins & $106(95 \%)$ \\
\hline Coronary angioplasty & $33(29 \%)$ \\
\hline Revascularization surgery & $15(13 \%)$ \\
\hline
\end{tabular}

$\mathrm{Cl}: 0.55-0.76 ; \mathrm{p}=0.01)$ - Figure $2 \mathrm{C}$. The prevalence of obstructive coronary artery disease had a tendency towards an increase according to the ascending TIMI tertiles (61\%, $69 \%, 86 \% ; p=0.056)$. Similarly, the TIMI score discriminated modestly patients with or without severe coronary artery disease (left main coronary artery or three-vessel disease) - area under the ROC curve of $0.66 ; 95 \% \mathrm{Cl}$ : $0.56-0.76$; $p=0.008$ - Figure 2D. That modest discriminatory capacity reflected in a lower prevalence of severe disease in the first GRACE tertile as compared with the two higher tertiles (15\%, $44 \%, 40 \%$, respectively; $p=0.10$ ).

\section{Discussion}

The present study does not confirm the initial hypothesis that the TIMI and GRACE risk scores predict accurately the presence and anatomical extension of coronary artery disease in patients with ACS.

In this study, coronary anatomy was described as a quantitative variable (Gensini score) and as a categorical variable (presence of obstructive or severe disease). In the 

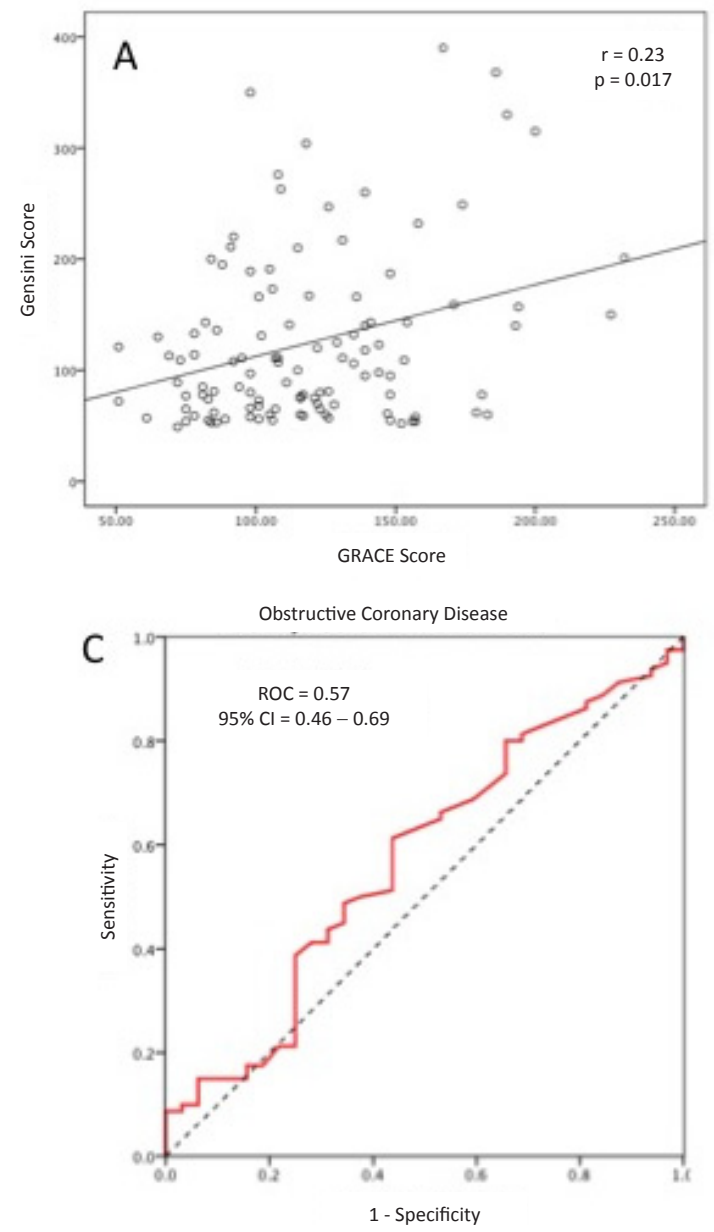
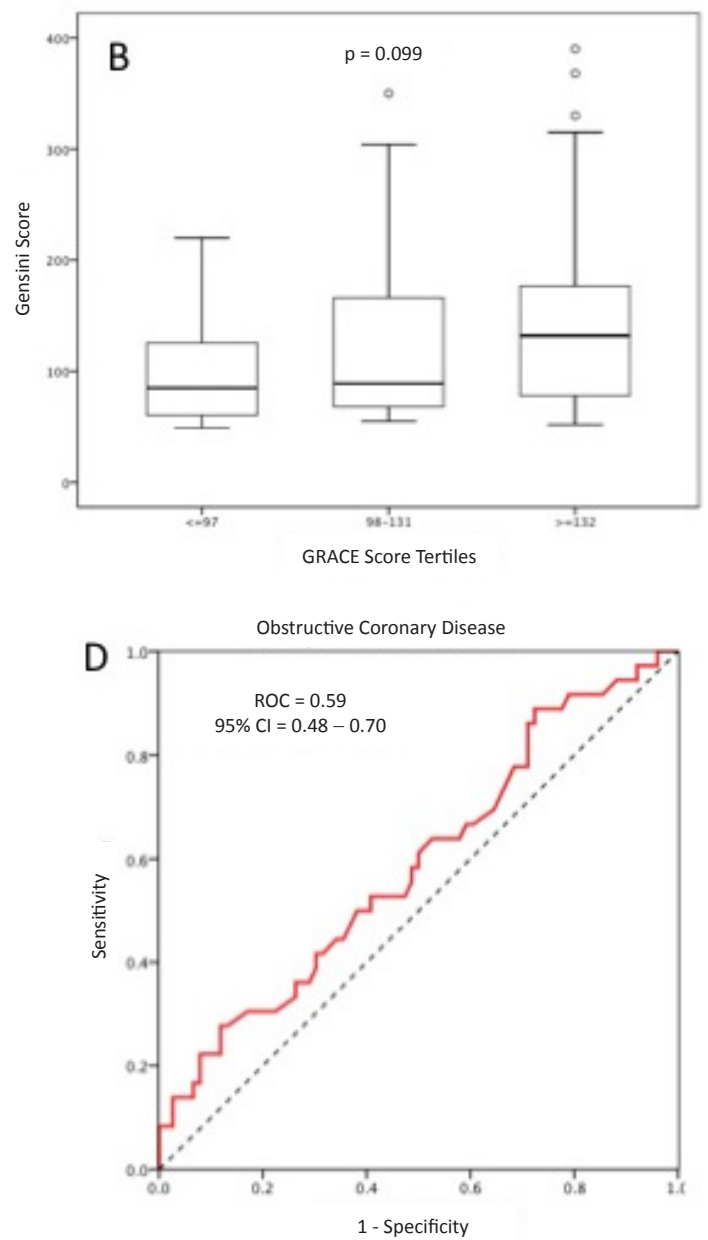

Figure 1 - Graph A represents the dispersion between the numerical value of the GRACE score and the numerical value of the Gensini score, evidencing a small correlation ( $r=0.23 ; p=0.017)$. Graph B compares the values of the Gensini score between the tertiles of the GRACE score, with no statistically significant association $(p=0.099)$. Graph $C$ represents the ROC curve of the GRACE score for detecting obstructive coronary artery disease, and graph $D$ represents the ROC curve of the GRACE score for detecting severe coronary artery disease, but they show no diagnostic accuracy.

first case, a statistically significant correlation of the coronary anatomy with both scores was observed. That tendency is confirmed by the dose-response gradient between the tertiles of the scores and the value of the Gensini score, statistically significant in the case of the TIMI score and borderline in the case of the GRACE score. Such analyses suggest that the prognostic information contained in the scores is partially mediated by the extension of coronary anatomy.

However, when analyzing the strength of the association of those scores with coronary anatomy, the results differ. First, the weak correlation $(r<0.30)$ with the Gensini score showed that the calibration of the prognostic scores to coronary anatomy is small. That is, using the regression formula for coronary artery disease extension, the difference between the predicted and the observed values of the Gensini score is large. Second, there is a reasonable overlap of the IQI of the Gensini score between the tertiles of the GRACE or TIMI scores. Third, the area under the ROC curve of the GRACE score is not statistically significant and that of the TIMI score, although statistically significant, shows modest accuracy in discriminating patients with obstructive disease or with severe disease (area $<0.70)$. Such data indicate that the prognostic scores do not play a good role as predictive models of coronary anatomy.

The prognosis of coronary artery disease is known to be directly influenced by the extension of arterial impairment ${ }^{12}$. How to explain that the prognostic models are not good predictive models of the anatomy? The response to that question originates from the fact that the disease anatomic extension represents only one of the several determinants of severity in a complex multivariate model, typical of most biological systems. That is, prognosis does not depend only on anatomy. Thus, those scores can predict risk, without necessarily predicting anatomy at the same proportion. For example, even having single-vessel disease, a patient can have an infarction with severe left ventricular dysfunction in the presence of proximal impairment of the descending anterior coronary artery, causing severe extensive ischemia. The severity of that patient would reflect in the hemodynamic 


\section{Original Article}

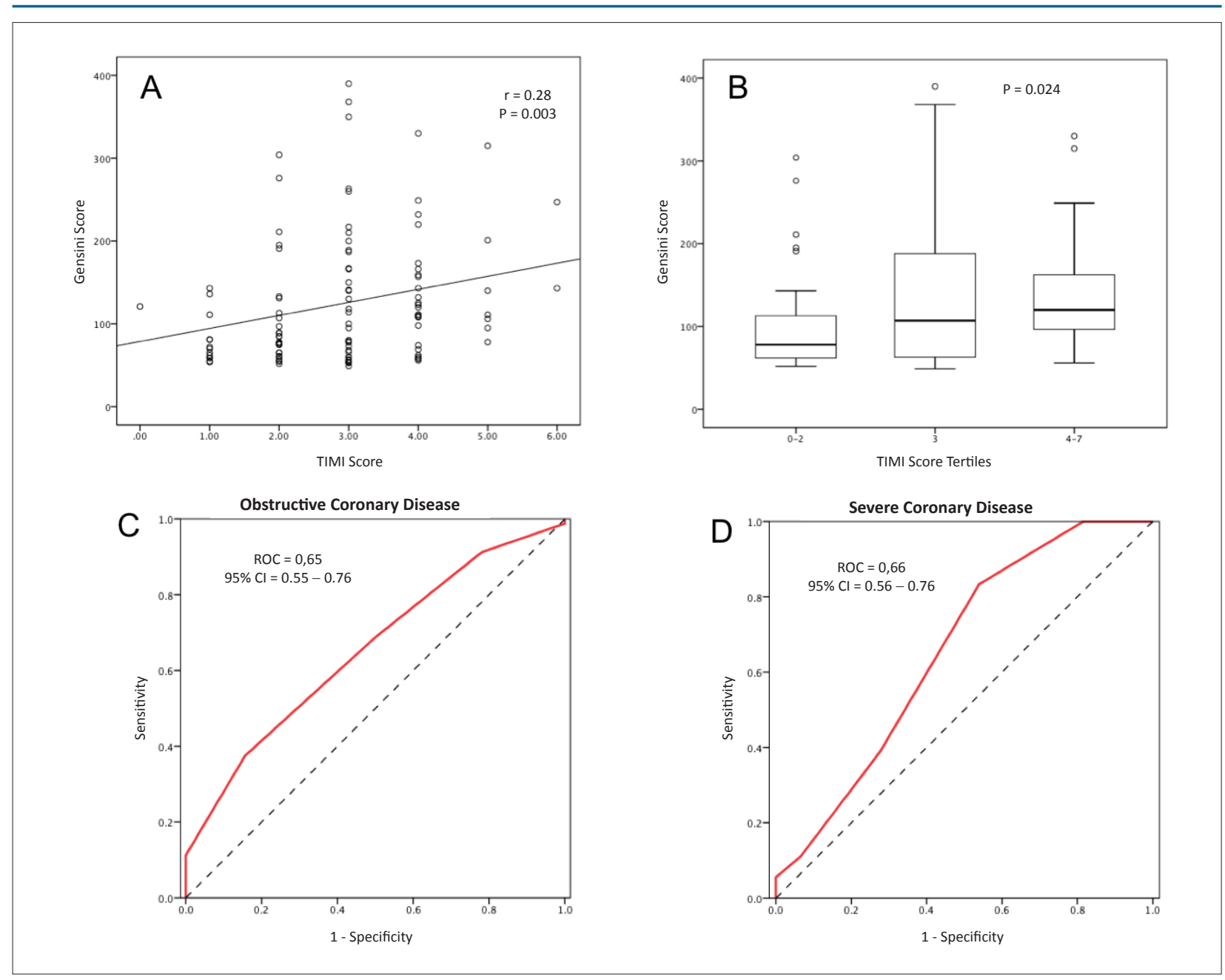

Figure 2 - Graph A represents the dispersion between the numerical value of the TIMI score and the numerical value of the Gensini score, evidencing a small correlation $(r=0.28 ; p=0.003)$. Graph B compares the values of the Gensini score between the tertiles of the TIMI score, evidencing an association between the Gensini value and the TIMI tertile $(p=0.024)$. Graph C represents the ROC curve of the TIMI score for detecting obstructive coronary artery disease, and graph D represents the ROC curve of the TIMI score for detecting severe coronary artery disease, evidencing modest diagnostic accuracy.

and electrocardiographic variables, and in the necrosis markers that constitute those scores.

The GRACE score has shown a greater prognostic value as compared with that of the TIMI score in different studies ${ }^{2,5,13}$. Paradoxically, the TIMI score has shown better prediction of the anatomy. This finding can be explained by the fact that the TIMI score considers some variables specifically related to the existence of coronary artery disease ${ }^{1}$. For example, one of the variables of TIMI score is the patient's previous knowledge about his/her obstructive coronary artery disease; in addition, previous aspirin use and number of risk factors, variables exclusive to the TIMI score, indicate more extensive coronary artery disease.

The practical implications of the results found should be discussed. Both scores are commonly used for the clinical decision of patients with ACS, aiming at defining the aggressiveness of the antithrombotic treatment and the either invasive or selective strategy of stratification ${ }^{14,15}$. That use is based on the established prognostic value of those scores and on the relationship between baseline risk and the magnitude of the benefit of certain strategies. It is worth noting that our results do not contradict the value of those scores for clinical decision. However, we should recognize that they would be more useful if they could predict coronary anatomy. This is due to the fact that the invasive strategy, applied to patients at medium to high risk according to those scores, is completed with a procedure of revascularization of the culprit artery. If the scores could better identify the presence of obstructive disease, they would identify patients to whom the invasive strategy would be more useful. Another example of usefulness would be the prediction of patients with extensive disease, preventing the administration of certain antiplatelet agents that would increase their risk of bleeding in an occasional early surgery.

Regarding originality, this is the first study describing the association between the GRACE score and coronary anatomy. Regarding the TIMI score, four studies have assessed that question ${ }^{4-7}$. All have reported a positive association 
between score value and coronary artery disease extension. However, we know that the association is not sufficient to ensure accuracy, which should be assessed by use of tests of calibration and discriminatory capacity of ill individuals versus healthy individuals (or severe disease versus non-severe disease). Because ours was the only study to perform that type of analysis, our conclusion contradict the TIMI score value in predicting anatomy, differing from the previous studies which were limited to demonstrating the presence of the association between the TIMI score and the number of arteries affected. Our data confirm the association with disease extension, but demonstrate that it is insufficient to provide satisfactory accuracy. In addition, of the studies cited, ours was the first to measure the coronary artery disease extension quantitatively by using the Gensini score.

This study had limitations, such as the selection bias regarding its target population, individuals with non-STelevation ACS. Ideally, we should assess the entire sample of patients meeting diagnostic criteria for that clinical condition. However, only $60 \%$ of the individuals with no previous surgery underwent coronary angiography, limiting the study sample. Although that is a characteristic inherent to most studies about coronary anatomy, it may cause a bias in the assessment of accuracy of risk scores regarding anatomy. In practice, patients with low scores tend to undergo coronary angiography less frequently. Such bias reduces the specificity of the risk score in recognizing patients without obstructive coronary artery disease. That is, those scores might identify a certain number of patients as having neither obstructive disease nor severe disease, but they were not computed in the analysis, because the reference standard (coronary angiography) was not performed. However, sensitivity can be overestimated, because the group of patients undergoing coronary angiography tends to have higher score values

\section{References}

1. Antman EM, Cohen M, Bernink PJ, McCabe CH, Horacek T, Papuchis G, et al. The TIMI risk score for unstable angina/non-ST elevation MI: a method for prognostication and therapeutic decision making. JAMA. 2000;284(7):835-42.

2. Yan AT, Yan RT, Tan M, Casanova A, Labinaz M, Sridhar K, et al. Risk scores for risk stratification in acute coronary syndromes: useful but simpler is not necessarily better. Eur Heart J. 2007;28(9):1072-8.

3. Granger CB, Goldberg RJ, Dabbous O, Pieper KS, Eagle KA, Cannon CP, et al.; Global Registry of Acute Coronary Events Investigators. Predictors of hospital mortality in the global registry of acute coronary events. Arch Intern Med. 2003;163(19):2345-53.

4. Lakhani MS, Qadir F, Hanif B, Farooq S, Khan M. Correlation of thrombolysis in myocardial infarction (TIMI) risk score with extent of coronary artery disease in patients with acute coronary syndrome. J Pak Med Assoc. 2010;60(3):197-200.

5. Mega JL, Morrow DA, Sabatine MS, Zhao XQ, Snapinn SM, DiBattiste PM, et al. Correlation between the TIMI risk score and high-risk angiographic findings in non-st-elevation acute coronary syndromes: observations from the Platelet Receptor Inhibition in Ischemic Syndrome Management in Patients Limited by Unstable Signs and Symptoms (PRISM-PLUS) trial. Am Heart J. 2005;149(5):846-50. more frequently. That phenomenon is called assessment bias $^{16}$. Thus, we avoided describing specifically sensitivity and specificity, choosing to analyze the area under the ROC curve, which would represent a joint analysis of those two diagnostic properties ${ }^{17}$.

Our second limitation is the relatively small sample size, which was partially overcome through the data analysis approach. First, the quantitative description of coronary artery disease (Gensini score) provided sufficient statistical power to the correlation analysis. Second, when describing the area under the ROC curve, the sample size was sufficient to ensure satisfactory precision. However, it is worth noting that future studies with a higher number of patients will provide more precision to our estimates.

In conclusion, the present study suggests that the TIMI and GRACE scores are not accurate tools to predict the coronary artery disease extension in patients with non-ST-elevation ACS. The usefulness of those scores for clinical reasoning should be limited to estimating the probability of recurring cardiovascular events.

\section{Potential Conflict of Interest}

No potential conflict of interest relevant to this article was reported.

\section{Sources of Funding}

This study was partially funded by Fundação de Amparo à Pesquisa da Bahia (FAPESB).

\section{Study Association}

This article is part of the thesis of master submitted by Carolina Esteves Barbosa, from Escola Bahiana de Medicina.

6. Garcia S, Canoniero M, Peter A, de Marchena E, Ferreira A. Correlation of TIMI risk score with angiographic severity and extent of coronary artery disease in patients with non-ST-elevation acute coronary syndromes. Am J Cardiol. 2004;93(7):813-6.

7. Ben Salem H, Ouali S, Hammas S, Bougmiza I, Gribaa R, Ghannem K, et al. [Correlation of TIMI risk score with angiographic extent and severity of coronary artery disease in non-ST-elevation acute coronary syndromes]. Ann Cardiol Angeiol (Paris). 2011;60(2):87-91.

8. Apple FS, Pearce LA, Smith SW, Kaczmarek JM, Murakami MM. Role of monitoring changes in sensitive cardiac troponin I assay results for early diagnosis of myocardial infarction and prediction of risk of adverse events. Clin Chem. 2009;55(5):930-7.

9. Apple FS, Quist HE, Doyle PJ, Otto AP, Murakami MM. Plasma 99th percentile reference limits for cardiac troponin and creatine kinase MB mass for use with European Society of Cardiology/American College of Cardiology consensus recommendations. Clin Chem. 2003;49(8):1331-6.

10. Khot UN, Jia G, Moliterno DJ, Lincoff AM, Khot MB, Harrington RA, et al. Prognostic importance of physical examination for heart failure in non-ST-elevation acute coronary syndromes: the enduring value of Killip classification. JAMA. 2003;290(16):2174-81. 


\section{Original Article}

11. Huang G, Zhao JL, Du H, Lan XB, Yin YH. Coronary score adds prognostic information for patients with acute coronary syndrome. Circ J. 2010;74(3):490-5.

12. Ringqvist I, Fisher LD, Mock M, Davis KB, Wedel H, Chaitman BR, et al. Prognostic value of angiographic indices of coronary artery disease from the Coronary Artery Surgery Study (CASS). J Clin Invest. 1983;71(6):1854-66.

13. Correia LC, Freitas R, Bittencourt AP, Souza AC, Almeida MC, Leal J, et al. [Prognostic value of GRACE scores versus TIMI score in acute coronary syndromes]. Arq Bras Cardiol. 2010;94(5):613-9.

14. Anderson JL, Adams CD, Antman EM, Bridges CR, Califf RM, Casey DE Jr, et al. 2011 ACCF/AHA Focused Update Incorporated Into the ACC/AHA 2007 Guidelines for the Management of Patients With Unstable Angina/
Non-ST-Elevation Myocardial Infarction: a report of the American College of Cardiology Foundation/American Heart Association Task Force on Practice Guidelines. Circulation. 2011;123(18):e426-579.

15. Hamm CW, Bassand JP, Agewall S, Bax J, Boersma E, Bueno H, et al. ESC Guidelines for the management of acute coronary syndromes in patients presenting without persistent ST-segment elevation: The Task Force for the management of acute coronary syndromes (ACS) in patients presenting without persistent ST-segment elevation of the European Society of Cardiology (ESC). Eur Heart J. 2011;32(23):2999-3054.

16. Whiting PF, Rutjes AW, Westwood ME, Mallett S, Deeks JJ, Reitsma JB, et al. QUADAS-2: a revised tool for the quality assessment of diagnostic accuracy studies. Ann Intern Med. 2011;155(8):529-36.

17. Obuchowski NA. Receiver operating characteristic curves and their use in radiology. Radiology. 2003;229(1):3-8 